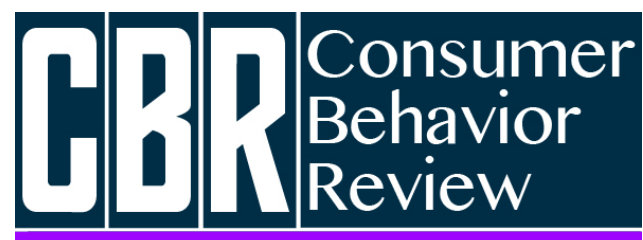

Revista Comportamento do Consumidor
Andrade, M. L., Leite, R. S. \& Sousa, D. C. (2019). Ética e o consumo de produtos falsificados. Consumer Behavior Review, 3(1) 1-17.
ISSN: 2526-7884

Editor: Prof. Dr. Marconi Freitas da Costa Email da revista: cbr@ufpe.br
Avaliação: Double blind review

Recebido: 21 de agosto de 2018

Aceito: 12 de março de 2019

\title{
ÉTICA E O CONSUMO DE PRODUTOS FALSIFICADOS
}

\author{
Matheus Lemos de Andrade \\ Ramon Silva Leite \\ Debora Cesar e Sousa
}

\begin{abstract}
Matheus Lemos de Andrade é Professor da Faculdade de Administração Milton Campos. E-mail: matheus@institutoolhar.com.br. Ramon Silva Leite é Professor do Programa de Pós-Graduação em Administração da PUC-Minas. E-mail: ramonsl@pucminas.br. Debora Cesar e Sousa é Graduada em Administração pela Faculdade de Administração Milton Campos. E-mail: dehcsousa@gmail.com.

Esta pesquisa teve fomento da CAPES Coordenação de Aperfeiçoamento de Pessoa de Nível Superior. Os autores agracedecem aos avaliadores pelos comentários para melhoria do artigo.
\end{abstract}

\begin{abstract}
Resumo
O consumo de falsificados é um fenômeno global de interesse de diversos pesquisadores do campo do marketing. 0 presente estudo objetivou explicar os elementos que influenciam a propensão ao consumo de falsificados, a partir das características demográficas e éticas do consumidor. Para tal foi realizado um survey com amostra de duzentos e três consumidores da Região Metropolitana de Belo Horizonte. Os resultados indicam que dentre as características demográficas avaliadas, o sexo e a renda familiar influenciam a propensão ao consumo de falsificados. Mulheres e consumidores com menor faixa de renda familiar apresentam maior propensão a tal comportamento. Sobre a influencia da ética, os resultados apontam que os consumidores que se mostram mais tolerantes às práticas de consumo legais, mesmo que eticamente questionáveis, apresentam maior propensão ao consumo de falsificados. Assim, sugere-se que campanhas que demonstrem que a compra de falsificações é ilegal podem contribuir para a redução deste comportamento.

Palavras-chave: Consumo de Falsificados, Ética no Marketing, Ética do Consumidor.
\end{abstract}

Esta obra está licenciada com uma Licença Creative Commons Atribuição 4.0 Internacional.

\section{INTRODUÇÃO}

0 consumo de falsificados é uma prática disseminada no Brasil (Casali, Paes, Machado, Medeiros \& Aragão, 2010; Andrade, Leite \&
Salvador, 2018) e no mundo (Solomon \& O`Brien, 1990; Prendergast et al., 2002; Gupta, Gould \& Pola, 2004; Vitel \& Muncy, 2005; Nill \& Shultz, 2009). Apesar da dificuldade de 
mensurar a magnitude deste mercado (Zimmerman \& Chaudhry, 2009), o Conselho Nacional de Combate à Pirataria e Delitos Contra a Propriedade Intelectual (CNPC, 2011) estimou que mais da metade dos softwares e produtos da indústria fonográfica consumidos no país são falsificações. 0 consumo de falsificados é um fenômeno global que em 2013 movimentou em torno de 461 bilhões de dólares em todo o mundo, o que representa $2,5 \%$ do comércio mundial daquele ano (OECD/EUIPO, 2016).

No campo do marketing, a maior parte dos estudos sobre o consumo de falsificados envolve a compreensão dos fatores que influenciam e motivam tal comportamento. Apesar de um substancial corpo de publicações, verifica-se que o conhecimento sobre os elementos que envolvem o consumo de produtos falsificados ainda se mostra fragmentado e incipiente devido às divergências entre seus resultados e à ausência de uma teoria amplamente aceita para sua explicação (Eisend \& Schuchert-Güler, 2006; Swami, ChamorroPremuzic, \& Furnham, 2009; Lee \& Yoo, 2009; Diniz, Leite \& Pinto, 2017).

Conforme Eisend e Schuchert-Güler (2006), Lee e Yoo (2009) e Staake, Thiesse e Fleisch (2009), os estudos de consumo que analisaram o consumo e a compra de falsificados revelam que este fenômeno é influenciado por diversos elementos, tais como: valores pessoais, atitudes, cultura, marca, percepção de risco, materialismo, legalismo, preço, status, dentre outros. Contudo, considerando o consumo de falsificados como um comportamento "desviante", tem-se a possibilidade de uma fecunda discussão proveniente do confronto entre elementos relacionados à ética dos consumidores e ao consumo de falsificados.

Sendo assim, o presente estudo partiu do framework teórico sobre ética no marketing (Hunt \& Vitell, 2006) e no consumo (Vitell \& Muncy, 2005) para responder à seguinte pergunta: Como as racionalidades e a percepção ética dos consumidores influenciam a propensão ao consumo de produtos falsificados? Em coerência com tal questão norteadora, foram estabelecidos quatro objetivos específicos para o estudo em tela: 1) Identificar, a partir das características demográficas, os segmentos de consumidores com maior e menor propensão ao consumo de falsificados; 2) Verificar a relação entre a propensão ao consumo de produtos falsificados $\mathrm{e}$ as racionalidades éticas deontológica e teleológica; 3) Verificar a relação entre as dimensões da escala ética de Vitell e Muncy (2005) e a propensão ao consumo de falsificados; 4) Propor um modelo teórico para explicação da propensão ao consumo de falsificados a partir da ética do consumidor e de suas características demográficas.

0 presente trabalho está estruturado em quatro seções, além desta introdução. 0 Referencial Teórico faz um resgate da literatura sobre o tema e se encerra com a proposição de um modelo teórico para explicação dos elementos que influenciam o consumo de falsificados. Em seguida apresenta-se a percurso metodológico do estudo empírico realizado. Já as duas últimas seções apresentam os resultados de tal estudo e as considerações finais, respectivamente.

\section{REFERENCIAL TEÓRICO}

Esta seção se inicia com a apresentação dos fundamentos teóricos relacionados à ética e faz um resgate dos estudos sobre o consumo de falsificados que analisaram este fenômeno a partir de tal arcabouço teórico. Sendo assim, ao final desta sessão foi possível propor dois modelos teóricos amparados na teoria sobre ética do consumidor, para explicação do fenômeno de consumo de falsificados.

\section{Ética}

Uma análise etimológica revela que o termo "ética" deriva do Grego Ethos, cujo significado pode ser entendido como "caráter", "costume" ou "modo de ser". Vazquez (2007) e Passos (2006) defendem que ética é um tema caro à Filosofia, cujo foco consiste na análise das condutas humanas. Em coerência com tais autores, Clavo (2008) afirma que a ética envolve as regras, os valores e as razões que os indivíduos estabelecem como referência para determinação de sua conduta. De modo complementar, Patrus-Pena (2012) defende que a ética se interessa pela discussão sobre os elementos que determinam se um ato ou comportamento é bom ou não e para quem. Sendo assim, tem-se que a ética está envolvida tanto na avaliação dos comportamentos 
próprios dos indivíduos, como também no modo como estes avaliam o comportamento de terceiros.

Deve-se enfatizar o caráter determinista (Burrel \& Morgan, 1979) assumido pelos pesquisadores sobre ética, no que se refere à concepção sobre a natureza humana dos indivíduos e grupos investigados. Conforme Morin (2007) e Vazquez (2007), sob o ponto de vista da ética, os indivíduos são tomados como seres capazes de compreender o seu lugar no mundo, agem de modo livre e racionalizado e primam pela solidariedade e responsabilidade.

Considerando que a ética envolve a análise das razões que legitimam os comportamentos humanos, Clavo (2008) afirma que a reflexão ética é uma prática intrínseca à sociedade, ou seja, é uma atividade cotidiana. Contudo, Morin (2007) chama atenção para os dilemas éticos, termo utilizado para caracterizar situações em que dois deveres legítimos se apresentam de modo antagônico aos indivíduos. Neste ponto, o autor demonstra que a ética traz à tona a conflituosa relação entre o altruísmo e o egocentrismo. Ainda segundo Morin (2007) a autoética é uma forma de pensamento que incorpora os sentimentos de honra, lealdade e responsabilidade, disciplinando o egocentrismo e desenvolvendo o altruísmo para consolidar a natureza social do ser humano, abrindo espaço para a vida em comunidade.

No que se refere ao consumo, considera-se que os mercados são espaços complexos, uma vez que promovem a relação entre seus atores, que podem apresentar interesses e códigos de conduta distintos. Sendo assim, é comum que os consumidores vivenciem dilemas éticos em atividades cotidianas de consumo (Tan, 2002; Vitell, 2003).

A Teoria Geral da Ética no Marketing (Hunt \& Vitell, 2006) propõe um modelo teórico para explicação das tomadas de decisão em situações que envolvem algum componente ético. Tal modelo propõe que ao identificar que uma decisão envolve algum aspecto ético, os indivíduos tendem a promover, em conjunto, dois tipos de avaliação. A racionalidade deontológica utiliza princípios, normas e valores determinados a priori, para avaliação ética em relação às alternativas identificadas pelo indivíduo. Já a racionalidade teleológica se baseia na avaliação das consequências de uma alternativa para determinar o quão desejável ela é. Este tipo de avaliação ética envolve três pontos básicos: a) a avaliação da probabilidade de consequências identificadas ocorrerem; b) a desejabilidade de cada consequência identificada; c) a importância atribuída pelo indivíduo, aos públicos que serão diretamente afetados (Hunt \& Vitell, 2006).

Considerando a conflituosa relação entre o altruísmo e o egocentrismo vivenciada pelos indivíduos ao longo de avaliações éticas, o estudo de Andrade et al. (2017) apresentou evidências de que as racionalidades teleológicas são mais assimiladas pelos indivíduos que atribuem maior importância à dimensão individualista dos valores (Schwartz et al., 2012), o que reforça o caráter egocêntrico da teleologia. Já os indivíduos que atribuem maior importância aos valores pessoais com foco social/coletivo assimilam melhor as racionalidades deontológicas.

Considerando que não haviam estudos amplos sobre ética no consumo, Vitell e Muncy (1992) propuseram uma escala que mensura a sensibilidade ética dos consumidores. Em sua versão mais atualizada (Vittel \& Muncy, 2005) esta escala apresenta trinta afirmativas, que dão origem a sete dimensões conceituais de comportamentos de consumo, que estruturam as análises derivadas de sua aplicação (Tabela 01).

Tabela 1

Dimensões da escala de ética do consumidor de Vitell e Muncy (2005)

\begin{tabular}{|c|c|}
\hline Categoria & Explicação \\
\hline $\begin{array}{l}\text { Beneficiamento Ativo } \\
\text { Ilegal (BAI) }\end{array}$ & $\begin{array}{l}\text { O consumidor se beneficia de uma ação ilegal, de modo deliberado, ou } \\
\text { seja, por iniciativa própria }\end{array}$ \\
\hline $\begin{array}{l}\text { Beneficiamento Passivo } \\
\text { (BP) }\end{array}$ & $\begin{array}{l}\text { O consumidor se beneficia por alguma prática antiética, mas que não } \\
\text { foi deflagrada deliberadamente por ele }\end{array}$ \\
\hline $\begin{array}{l}\text { Beneficiamento Ativo } \\
\text { Legal (BAL) }\end{array}$ & $\begin{array}{l}\text { O consumidor se beneficia de modo deliberado por uma ação legal, } \\
\text { mas moralmente questionável }\end{array}$ \\
\hline
\end{tabular}




\begin{tabular}{l|c}
\hline $\begin{array}{l}\text { Beneficiamento Sem } \\
\text { Danos (BSD) }\end{array}$ & $\begin{array}{c}\text { O consumidor se beneficia por alguma prática questionável, seja legal } \\
\text { ou ilegal, mas que não gera danos diretos a nenhum público }\end{array}$ \\
\hline $\begin{array}{l}\text { Downloading e Compra } \\
\text { de Falsificados (DCF) }\end{array}$ & $\begin{array}{c}\text { Comportamentos de compra de produtos falsificados e de desrespeito } \\
\text { aos direitos autorais }\end{array}$ \\
\hline Reciclagem (REC) & $\begin{array}{c}\text { Comportamentos relacionados com a preocupação e preservação do } \\
\text { meio ambiente }\end{array}$ \\
\hline Fazer o Bem (FB) & $\begin{array}{c}\text { Comportamentos cujo consumidor abre mão de benefícios próprios } \\
\text { em favor de terceiros. Envolve algum grau de altruísmo. }\end{array}$ \\
\hline Fonte: Elaborado pelos autores, adaptado de Vitell e Muncy (2005)
\end{tabular}

Como contribuição, os autores demonstram que os consumidores são mais tolerantes com algumas ações potencialmente antiéticas do que com outras. Dentre as categorias avaliadas, temse que os comportamentos cujo consumidor se beneficia ativamente por uma ação ilegal (BAI) são os que recebem os maiores índices de reprovação. Em seguida, o beneficiamento passivo (BP), por gerar consequências negativas a terceiros, é identificado como a segunda categoria mais condenada. Em análise aos resultados destas duas categorias, os autores afirmam que as pessoas tendem a aceitar mais os comportamentos passivos do que os ativos, mesmo que em ambos os casos tais comportamentos sejam considerados errados (Vitell \& Muncy, 1992).

A terceira categoria de ações questionáveis em ordem de reprovação são aquelas executadas de modo deliberado, que geram algum tipo de benefício para o consumidor, mas que não envolvem nenhuma ilegalidade (BAL). Por serem legais, há uma maior aceitação de tais práticas, mesmo que majoritariamente sejam percebidas como antiéticas. Já as práticas questionáveis avaliadas pela escala MuncyVitell com menor índice de reprovação são aquelas que não geram consequências negativas diretas para terceiros (BSD), assim como as relativas à prática de Downloads não autorizados e a compra de falsificados (DCF). Coerente com o pensamento teleológico, como não há a percepção de resultados maléficos, há a tendência de que sejam toleradas (Vitell \& Muncy, 1992).

No que se refere às dimensões positivas da escala proposta por Vitell e Muncy (2005), os resultados indicam que os comportamentos que compõem a dimensão Fazer o Bem (FB) são considerados mais corretos do que os relacionados a Reciclagem (REC).

\section{Ética e o Consumo de Falsificados}

São raros os estudos que investigaram a relação entre o consumo de falsificados e a ética do consumidor. Nill e Shultz (1996) afirmam que a compra de produtos falsificados é um típico dilema ético e que os consumidores utilizam os julgamentos deontológico e teleológico para avaliar a compra de tais produtos. Considerando o pensamento deontológico, a compra de falsificados poderia ser facilmente combatida caso os consumidores percebessem os problemas morais imbricados no seu consumo, tal como o desrespeito aos direitos autorais. Os autores sugerem que a compra de falsificados poderia ser associada a um comportamento desonesto, tal como "roubar". No que se refere ao pensamento teleológico, os autores sugerem que os consumidores tendem a verificar a similaridade entre os produtos falsificados e os originais. Quanto mais diferentes forem tais produtos, considerando aparência, funcionalidade e durabilidade, menor seria a propensão ao consumo de falsificados.

Em contraposição a Nill e Shultz (1996), Costa e Sant'Anna (2008) consideram que em várias culturas o consumo de falsificados é fortemente disseminado e não gera interpretações negativas, o que poderia levar ao entendimento de que o seu consumo é um comportamento ético. Além disto, considerando o poder econômico e o controle exercido pelas grandes empresas detentoras das marcas líderes de mercado, seria plausível considerar que tanto para as empresas locais, quanto para os consumidores de baixa renda, o consumo de falsificados poderia ser compreendido como uma forma de inclusão social e de fortalecimento das economias subdesenvolvidas.

Em análise ao trabalho de Vitell e Muncy (1992), verifica-se que as afirmativas que 
tratam de atividades de consumo que ferem direitos autorais foram agrupadas na dimensão Beneficiamento Sem Danos (BSD). Como esta categoria é a que gera menor índice de reprovação, entende-se que o consumo de falsificados seja um comportamento socialmente aceito, provavelmente pela ausência de percepção dos danos diretos causados por tal conduta.

Penz e Stöttinger (2005) analisaram o consumo de falsificados entre os Australianos. Segundo os autores, a percepção ética (Fullerton's et al., 1996) está relacionada com a atitude em relação aos falsificados. Sendo assim, quanto mais ético é o consumidor, maior é sua preocupação em ser descoberto com tais produtos.

Alguns estudos nacionais analisaram o consumo de falsificados e sua relação com elementos éticos. Rabello, Leite e Gonçalves Filho (2016) apontam para uma maior propensão ao consumo de falsificados entre as classes sociais mais altas (A e B). Sobre as racionalidades éticas, consumidores que concordam com justificativas deontológicas contrárias ao consumo de falsificados apresentam menor propensão à sua compra. Já aqueles que concordam com as justificativas teleológicas favoráveis ao consumo de falsificados tendem a apresentar maior propensão a tal comportamento.

Santos, Gonçalves Filho e Falce (2017) defendem que quanto maior o nível ético do consumidor (Vitell \& Muncy, 2005), menor é a intenção de consumir falsificados. Sobre as características demográficas, o estudo indica que quanto maior a escolaridade, a renda e a idade do consumidor, menor é a intenção de adquirir produtos falsificados.

Casali, Paes, Machado, Medeiros e Aragão (2010) investigaram a relação entre ética e o consumo de softwares piratas por meio da aplicação da escala de ética do consumidor de Vitell e Muncy (2005). Os autores identificaram que piratear softwares não é percebido predominantemente como algo antiético, corroborando com os achados de outros estudos (Solomon \& O’Brien, 1990; Prendergast et al., 2002; Vitel \& Muncy, 2005; Gupta, Gould \& Pola, 2004; Nill \& Shultz, 2009). Reforçando os achados de Santos, Gonçalves Filho e Falce (2017), os resultados também revelam que os entrevistados são mais tolerantes às práticas questionáveis de consumo que não geram consequência negativas diretas junto a outros públicos (BSD) e as que, apesar de serem questionáveis, não envolvem nenhum ato ilegal (BAL).

\section{Proposições e Construção do Modelo Teórico}

Considerando os objetivos deste empreendimento científico e a análise da literatura sobre o tema, tem-se que o entendimento sobre a relação entre ética do consumidor e consumo de falsificados ainda carece de maior escrutínio. No que se refere às características demográficas, verifica-se uma certa divergência entre os resultados de Rabello, Leite e Gonçalves Filho (2016) e o trabalho de Santos, Gonçalves Filho e Falce (2017). Enquanto o primeiro estudo aponta para o maior consumo de falsificados entre as camadas sociais mais elitizadas, o segundo aponta para a direção contrária. Além disto, enquanto no primeiro não houve associação entre idade e consumo de falsificados, no segundo esta relação foi considerada significativa.

Sendo assim, no que se refere às características demográficas, o presente estudo propõe que hajam diferenças significativas do índice médio de propensão ao consumo de falsificados, quando se analisam segmentos de consumidores estratificados por sexo, idade, escolaridade e renda familiar.

Sobre as racionalidades éticas, verifica-se a fragilidade dos achados de Rabello, Leite e Gonçalves Filho (2016), uma vez que as afirmativas utilizadas para mensurar as racionalidades deontológicas e teleológicas já apresentavam um posicionamento favorável (deontológica) e contrário (teleológica) ao consumo de falsificados, o que inviabiliza a possibilidade de fazer inferências sobre relações entre tais racionalidades e qualquer aspecto relacionado ao consumo de falsificados.

Considerando que o presente estudo irá mensurar o quanto cada indivíduo assimila as racionalidades deontológicas e teleológicas, as proposições relacionadas ao segundo objetivo do presente estudo apontam para a existência de relações significativas entre as racionalidades éticas e a propensão ao consumo de falsificados. Adotando-se as proposições de 
Nill e Shultz (1996), as hipóteses estabelecidas preveem a existência de uma relação positiva entre a ética teleológica e a propensão ao consumo de falsificados, bem como uma relação negativa entre tal construto e a racionalidade deontológica.

Já em relação ao terceiro objetivo, considerando os achados de Casali et al. (2010) e Santos, Gonçalves Filho e Falce (2017), o presente estudo propõe que haja uma relação negativa entre a sensibilidade ética do consumidor e a propensão ao consumo de falsificados. Além disto, espera-se que as cinco dimensões de comportamentos eticamente questionáveis apresentem relações positivas com a propensão ao consumo de falsificados, ou seja, espera-se que quanto mais o consumidor considera que os comportamentos listados na escala de Vitell e Muncy (2005) não sejam errados, maior seja sua propensão ao consumo de falsificados. Considerando o que foi exposto, a Tabela 02 apresenta as hipóteses estabelecidas para o presente empreendimento científico.

Considerando o objetivo de propor um modelo teórico para explicação da propensão ao consumo de falsificados a partir de elementos éticos, adotou-se a estratégia de modelos concorrentes sugerida por Hair et al. (2009). De acordo com os autores, esta estratégia se diferencia da abordagem confirmatória da modelagem de equações estruturais e basicamente prevê a construção de dois ou mais modelos teóricos que serão testados e comparados pelo pesquisador. Considerando que todas as propostas de modelos devem ser respaldadas pela teoria, o modelo com melhores resultados de ajuste é assumido como a melhor forma de descrição da teoria em análise. A Figura 01 apresenta a ilustração de dois modelos distintos. Enquanto o primeiro (Figura 01-A) estabelece relações entre as dimensões éticas de consumo da escala de Vitell e Muncy (2005) e a propensão ao consumo de falsificados, o segundo modelo (Figura 01-B) prevê que a sensibilidade ética terá maior poder de explicação em relação à propensão ao consumo de falsificados. Sendo assim, diferente do modelo "A", o modelo "B" trata a sensibilidade ética do consumidor como um construto único, sem diferenciar suas dimensões.

\section{Tabela 02}

Hipóteses postuladas

\begin{tabular}{l|l}
\hline No. & \multicolumn{1}{c}{ Hipóteses } \\
\hline H1 & Há diferenças significativas entre homens e mulheres no que se refere à média de PCF \\
\hline H3 & $\begin{array}{l}\text { à má diferenças significativas entre consumidores de diferentes faixas etárias no que se refere } \\
\text { se refere à média de PCF }\end{array}$ \\
\hline H4 & $\begin{array}{l}\text { Há diferenças significativas entre consumidores de diferentes níveis de renda familiar no } \\
\text { que se refere à média de PCF }\end{array}$ \\
\hline H5 & Quanto mais o consumidor assimila a racionalidade deontológica, menor é a PCF \\
\hline H6 & Quanto mais o consumidor assimila a racionalidade teleológica, maior é a PCF \\
\hline H7 & Quanto mais eticamente sensível é o consumidor, menor é a PCF \\
\hline H8 & $\begin{array}{l}\text { Quanto mais o consumidor considera que os comportamentos de Beneficiamento Ativo } \\
\text { Ilegal (BAI) não são errados, maior é a PCF }\end{array}$ \\
\hline H9 & $\begin{array}{l}\text { Quanto mais o consumidor considera que os comportamentos de Beneficiamento Passivo } \\
\text { (BP) não são errados, maior é a PCF }\end{array}$ \\
\hline H10 & $\begin{array}{l}\text { Quanto mais o consumidor considera que os comportamentos de Beneficiamento Ativo } \\
\text { Legal (BAL) não são errados, maior é a PCF }\end{array}$ \\
\hline H11 & $\begin{array}{l}\text { Quanto mais o consumidor considera que os comportamentos de Beneficiamento Sem } \\
\text { Danos (BSD) não são errados, maior é a PCF }\end{array}$ \\
\hline H12 & $\begin{array}{l}\text { Quanto mais o consumidor considera que os comportamentos de Downloading e Compra de } \\
\text { Falsificados (DCF) não são errados, maior é a PCF }\end{array}$ \\
\hline
\end{tabular}

Fonte: Elaborado pelos autores 


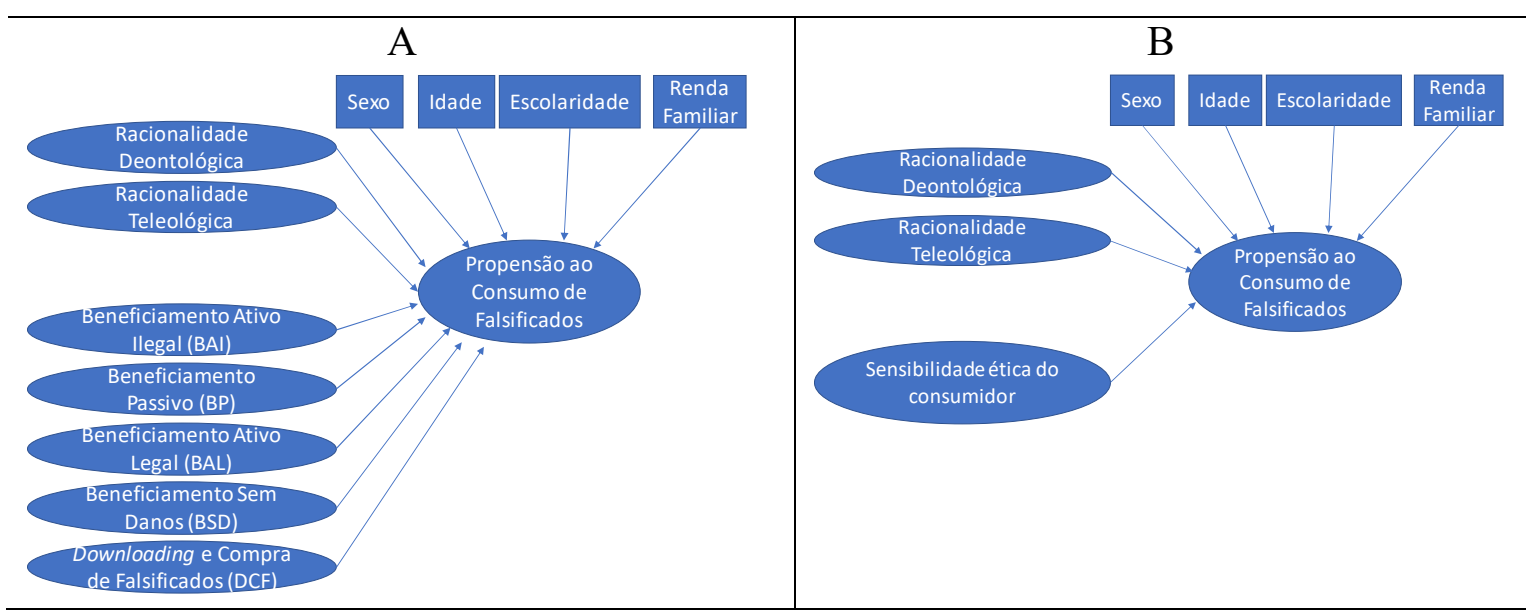

Fonte: Elaborado pelos autores

Figura 01: Modelos teóricos propostos

\section{METODOLOGIA}

0 presente empreendimento científico se caracteriza por uma abordagem descritiva, com aplicação de método quantitativo e da técnica Survey de Opinião (Malhotra \& Birks, 2007). A amostra foi coletada por meio de um questionário eletrônico estruturado, programado no aplicativo SurveyMonkey. Os links do questionário foram enviados aos contatos pessoais dos pesquisadores, aos quais foi solicitado o reenvio para suas respectivas redes de contato. Sendo assim, tal amostra pode ser considerada Não Probabilística do tipo por Conveniência, com o emprego da estratégia Bola de Neve (Malhotra \& Birks, 2007). Em fase preliminar, de pré-teste, o instrumento de coleta de dados foi aplicado junto a 14 elementos. A coleta ocorreu entre julho e agosto de 2017 e envolveu consumidores residentes nas Região Metropolitana de Belo Horizonte.

No que se refere à mensuração dos construtos teóricos, para avaliação das racionalidades éticas adotou-se a escala utilizada por Burns e Kieker (1995), com intervalo de 11 pontos (escala Likert de 0 a 10), já traduzida e validada no Brasil (Andrade et al., 2017). A escala ética do consumidor (Vitell \& Muncy, 2005) foi aplicada utilizando-se somente os itens que compõem as dimensões de comportamentos eticamente questionáveis (BAI; BP; BAL; BSD; DCF), com intervalo de 07 pontos (escala Likert de 1 a 7). Os itens relacionados às dimensões Reciclagem (REC) e Fazer o Bem (FB) foram excluídos por não se inserirem na discussão proposta para o presente estudo. Para tal, utilizou-se a versão já traduzida e validada no Brasil por Santos, Gonçalves Filho e Falce (2017). Já para a mensuração da propensão ao consumo de falsificados, utilizou-se uma lista de sete produtos, sendo perguntado aos entrevistados qual a possibilidade dele(a) vir a comprar uma versão falsificada, caso fosse de boa qualidade e apresentasse bom preço. Tal medição foi realizada a partir das proposições de Furnham e Valgeirsson (2007) e utilizou a escala Likert com 08 pontos (de 0 a 7 ).

Sobre o tratamento dos dados, adotou-se uma abordagem voltada para a teoria (Nunnaly \& Bernstein, 1994), sendo utilizados procedimentos da Análise Fatorial Confirmatória, especificamente aqueles relacionados à eliminação de itens cujas cargas fatoriais se mostraram baixas, a ponto de comprometer a confiabilidade ou a variância média extraída dos construtos operacionalizados (Ringle, Silva \& Bido, 2014). Tal procedimento foi realizado por meio do Software SmartPLS (Ringle et al., 2015), uma vez que se constitui como a primeira etapa da Modelagem de Equações Estruturais (Hair et al., 2009). 0 Apêndice 01 apresenta os itens que compõem as escalas utilizadas e identifica aqueles que permaneceram após a fase de avaliação das mensurações.

\section{RESULTADOS}

\section{Análise exploratória preliminar}

Apesar do esforço de coleta de dados ter resultado em 258 respostas, a análise exploratória do banco de dados serviu para eliminar os casos inapropriados. Sendo assim, 
seguindo os procedimentos adotados por Andrade et al., (2017), inicialmente foram eliminados os casos em que um respondente apresentou a mesma resposta para mais de $76 \%$ dos itens que compõem as escalas de Alinhamento Ético (Burns \& Kiecker, 1995) e de Ética do Consumidor (Vitell \& Muncy, 2005). Este procedimento levou à exclusão de 04 (quatro) respondentes. Em seguida eliminou-se os questionários parcialmente preenchidos, o que resultou na exclusão de mais cinquenta e um casos. Seguindo as orientações de Costa (2011), avaliou-se a existência de dados ausentes. Como a coleta foi realizada por meio de sistema eletrônico, nenhum respondente que completou o questionário deixou questões sem resposta, uma vez que o sistema exigia o preenchimento de todos os campos. Sendo assim, tem-se que a amostra final é formada por 203 (duzentos e três) respondentes.

Em relação ao número de respondentes, verificou-se se a amostra encontrada atendia aos requisitos apresentados por Costa (2011), que recomenda ao menos 05 (cinco) medições para cada variável que compõe a escala a ser testada. Considerando que a maior escala do questionário possui 23 variáveis, tem-se que a amostra final é adequada para analisar os construtos teóricos de interesse da presente investigação.

\section{Avaliação dos vieses de método comum}

Visando minimizar a incidência dos vieses de método comum (Podsakoff, MacKenzie \& Podsakoff, 2003), aos entrevistados foi dada total liberdade para responder ao questionário, uma vez que foram informados que não haviam repostas certas ou erradas para nenhuma das perguntas. Também foi garantido o anonimato dos respondentes, assim como os itens que compõem as escalas foram aplicados de modo randomizado. Visando avaliar a incidência de tal viés, aplicou-se o teste de fator único de Harmam, o qual apontou para a ausência de tais desvios, uma vez que 14 (quatorze) fatores com auto-valores maiores que 1 emergiram a partir da solução não rotacionada e que a capacidade explicativa deste conjunto de fatores foi de $63,5 \%$ da variância dos dados.

\section{Caracterização do público investigado}

Os resultados demonstram que os entrevistados apresentam maior concentração de mulheres (64,5\%), com idade entre 18 e 30 anos $(50,7 \%)$ e renda familiar acima de 08 salários mínimos (50,3\%). A grande maioria $(91,1 \%)$ possui escolaridade superior incompleta, completa ou são pós-graduados (Tabela 03).

Tabela 03

Caracterização da Amostra

\begin{tabular}{clcc}
\hline & & F & $\%$ \\
\hline \multirow{5}{*}{ Sexo } & Masculino & 72 & $35,5 \%$ \\
& Feminino & 131 & $64,5 \%$ \\
\hline \multirow{5}{*}{ Idade } & Até 17 anos & 5 & $2,5 \%$ \\
& De 18 a 30 anos & 103 & $50,7 \%$ \\
& De 31 a 45 anos & 53 & $26,1 \%$ \\
& De 46 a 59 anos & 35 & $17,2 \%$ \\
& 60 anos ou mais & 7 & $3,4 \%$ \\
\hline \multirow{5}{*}{ Renda Familiar } & Até 02 salários mínimos & 7 & $3,4 \%$ \\
& Entre 02 e 04 salários mínimos & 32 & $15,8 \%$ \\
& Entre 04 e 08 salários mínimos & 62 & $30,5 \%$ \\
& Entre 08 e 15 salários mínimos & 47 & $23,2 \%$ \\
& Mais de15 salários mínimos & 55 & $27,1 \%$ \\
\hline \multirow{5}{*}{ Escolaridade } & Fundamental (completo e & 1 & $0,5 \%$ \\
& incompleto) & & \\
& Ensino Médio (completo e & 17 & $8,4 \%$ \\
& incompleto) & & \\
& Superior incompleto & 70 & $34,5 \%$ \\
& Superior Completo & 62 & $30,5 \%$ \\
& Pós-Graduado & 53 & $26,1 \%$ \\
\hline
\end{tabular}

Fonte: Dados da pesquisa 


\section{Propensão ao consumo de falsificados}

Sobre a propensão ao consumo de falsificados, seguindo os procedimentos adotados por Furnham e Valgeirsson (2007), inicialmente avaliou-se como se comportaram os dados a partir dos procedimentos da Análise Fatorial Exploratória. Sendo assim, ao analisar a solução rotacionada (Varimax) a partir do método de extração de Componentes Principais, verificou-se que a solução encontrada apresentou dois fatores com auto-valores maiores que 1 . Considerando que tal solução explica 58,1\% da variação dos dados, tem-se que a mesma pode representar a propensão ao amplo, não limitada a nenhuma categoria específica de produtos. Vale mencionar que o primeiro fator encontrado (explicação de $39,1 \%$ ) é formado pelos itens que se relacionam com o consumo de pirataria (DVD's, Softwares e
CD de música). Já o segundo fator (explicação de $19,0 \%$ reflete o consumo de produtos falsificados de diferentes categorias. São elas: roupas, relógios, perfumes e óculos escuros

Considerando que a escala de Propensão ao Consumo de Falsificados foi avaliada a partir do intervalo que varia de 0 (zero) a 7 (sete) pontos, tem-se que índices médios menores 3,5 podem ser considerados com indicadores de baixa propensão. De modo similar, índices acima do ponto médio da escala podem ser interpretados como indicativos de alta propensão ao consumo de falsificados. Em análise ao Gráfico 1, nota-se que o índice médio encontrado para todos os itens $(2,7)$ aponta para uma baixa propensão geral ao consumo de falsificados. Enquanto DVD`s obtiveram a maior propensão ao consumo de falsificados $(4,0)$, perfumes é a categoria onde ocorre a menor propensão $(0,8)$.

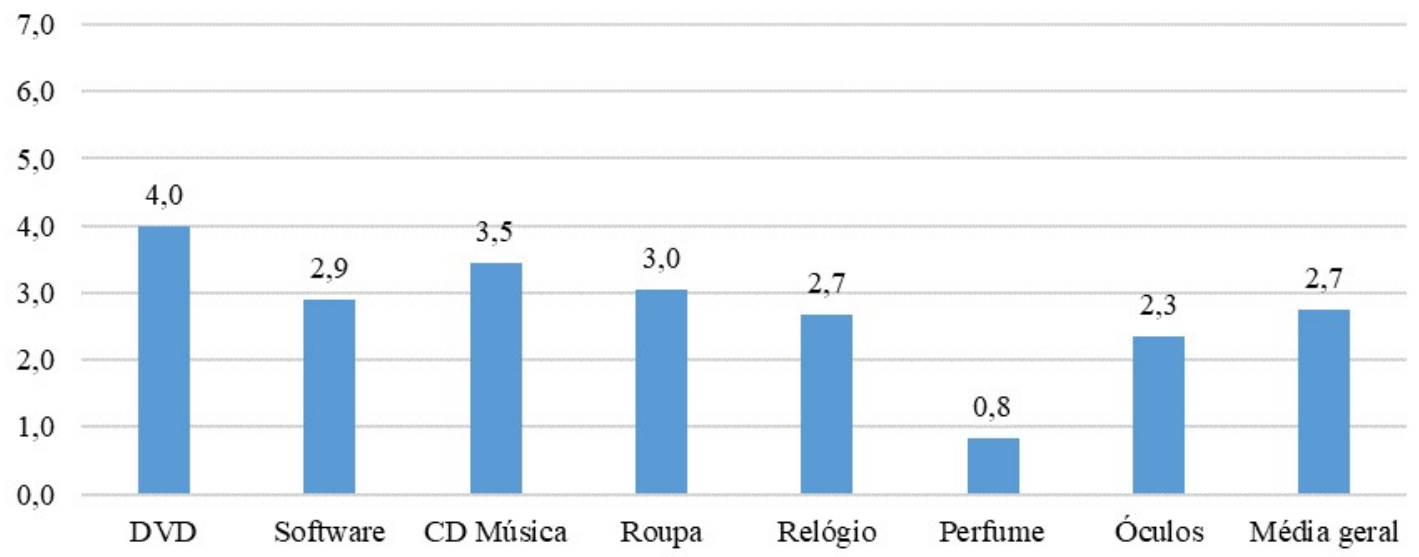

Fonte: Dados da Pesquisa

Gráfico 1: Propensão ao consumo de falsificados

\section{Características demográficas e propensão ao consumo de falsificados}

Considerando o objetivo de identificar os grupos com maior e menor propensão ao consumo de falsificados, foram utilizados testes não paramétricos de comparação de médias entre amostras independentes (Malhotra \& Birks, 2007). Desta forma verifica-se que todas as características demográficas avaliadas se mostram relevantes em relação à média da propensão ao consumo de falsificados, o que permite considerar a confirmação das hipóteses H1, H2, H3, H4. Tais diferenças se mostram estatisticamente significativas ao nível de até 95\% de confiança, exceto no caso do nível de escolaridade, cuja significância é confirmada ao nível de confiabilidade de $90 \%$ (Tabela 05).

Tabela 05

Testes de comparação de média

\begin{tabular}{c|l|c|c|c}
\hline Hipótese & \multicolumn{1}{|c|}{ Variável } & Teste Utilizado & Sig & Decisão \\
\hline H1 & Faixa etária & Kruskal-Wallis & 0,041 & Rejeitar hipótese nula \\
\hline H2 & Sexo & Mann-Whitney & 0,019 & Rejeitar hipótese nula \\
\hline
\end{tabular}




\begin{tabular}{c|l|l|l|l}
\hline H3 & Escolaridade & Kruskal-Wallis & 0,056 & Rejeitar hipótese nula \\
\hline H4 & Renda Familiar & Kruskal-Wallis & 0,000 & Rejeitar hipótese nula \\
\hline
\end{tabular}

Ao analisar as médias de propensão ao consumo de falsificados por estrato, verifica-se que exceto aqueles que possuem renda familiar de até 02 salários mínimos, quanto maior a renda familiar, menor é a propensão. Em relação à escolaridade, os resultados se mostram similares, uma vez que exceto o segmento com menor nível educacional, quanto maior a escolaridade, menor é a propensão ao consumo de falsificados. Os resultados também indicam que os jovens (18 a 29 anos) e as mulheres compõem os segmentos com maior propensão ao consumo de falsificações (Tabela 06).

Tabela 06

Média de Propensão ao Consumo de Falsificados, por segmento

\begin{tabular}{|c|c|c|c|}
\hline \multirow{2}{*}{ Critério } & \multirow{2}{*}{ Segmento } & \multicolumn{2}{|c|}{$\begin{array}{c}\text { Propensão ao Consumo de } \\
\text { Falsificados }\end{array}$} \\
\hline & & Média & $\begin{array}{l}\text { Desvio } \\
\text { Padrão }\end{array}$ \\
\hline \multirow{5}{*}{ Faixa Etária } & Até 17 anos & 2,0 & 0,865 \\
\hline & De 18 a 30 anos & 3,1 & 1,681 \\
\hline & De 31 a 45 anos & 2,5 & 1,868 \\
\hline & De 46 a 59 anos & 2,6 & 1,298 \\
\hline & 60 anos ou mais & 1,6 & 1,108 \\
\hline \multirow{2}{*}{ Sexo } & Masculino & 2,4 & 1,463 \\
\hline & Feminino & 3,0 & 1,747 \\
\hline \multirow{4}{*}{ Escolaridade } & $\begin{array}{l}\text { Fundamental - } \\
\text { Médio }\end{array}$ & 2,8 & 1,239 \\
\hline & $\begin{array}{l}\text { Superior } \\
\text { incompleto }\end{array}$ & 3,0 & 1,616 \\
\hline & Superior completo & 2,8 & 1,813 \\
\hline & Pós-graduado & 2,2 & 1,632 \\
\hline \multirow{5}{*}{$\begin{array}{l}\text { Renda } \\
\text { Familiar }\end{array}$} & Até 02 SM & 2,8 & 1,186 \\
\hline & De 02 a 04 SM & 3,4 & 1,730 \\
\hline & De 04 a 08 SM & 3,2 & 1,665 \\
\hline & De 08 a 15 SM & 2,7 & 1,572 \\
\hline & $15 \mathrm{SM}$ ou mais & 1,9 & 1,467 \\
\hline
\end{tabular}

Fonte: Dados da pesquisa

\section{Avaliação dos modelos de mensuração}

Visando a concretização dos demais objetivos propostos, optou-se pela utilização da técnica de Modelagem de Equações Estruturais a partir da abordagem dos Mínimos Quadrados Parciais. Tal escolha se justifica pela ausência da exigência da normalidade dos dados, bem como pela possibilidade de utilização de variáveis categóricas no modelo (Ringle, Silva \& Bido,
2014), como no caso das características demográficas. A Tabela 07 apresenta os resultados da avaliação da qualidade das mensurações dos dois modelos a serem testados, considerando suas medidas finais, após o processo de exclusão de itens com baixa carga fatorial. 
Tabela 07

Qualidade das mensurações

\begin{tabular}{|c|c|c|c|c|c|}
\hline \multirow[t]{2}{*}{ Modelo } & \multirow[t]{2}{*}{ Construto Teórico } & \multirow{2}{*}{$\begin{array}{l}\text { № } \\
\text { Itens }\end{array}$} & \multicolumn{2}{|c|}{$\begin{array}{l}\text { Confiabilidade } \\
\text { Composta }\end{array}$} & \multirow[t]{2}{*}{ AVE } \\
\hline & & & A & B & \\
\hline \multirow{5}{*}{ Modelo A } & Beneficiamento Ativo Ilegal (BAI) & 02 & 0,773 & - & 0,632 \\
\hline & Beneficiamento Passivo (BP) & 03 & 0,760 & - & 0,521 \\
\hline & Beneficiamento Ativo Legal (BAL) & 02 & 0,760 & - & 0,613 \\
\hline & Beneficiamento Sem Danos (BSD) & 02 & 0,828 & - & 0,707 \\
\hline & $\begin{array}{l}\text { Downloading e Compra de Falsificados } \\
\text { (DCF) }\end{array}$ & 04 & 0,857 & - & 0,600 \\
\hline Modelo B & Sensibilidade ética & 05 & - & 0,852 & 0,537 \\
\hline \multirow{3}{*}{$\begin{array}{l}\text { Comum aos } \\
\text { modelos A e B }\end{array}$} & Racionalidade Deontológica & 02 & 0,732 & 0,732 & 0,579 \\
\hline & Racionalidade Teleológica & 03 & 0,752 & 0,752 & 0,507 \\
\hline & Propensão ao Consumo de Falsificados & 04 & 0,837 & 0,837 & 0,566 \\
\hline
\end{tabular}

Fonte: Dados da pesquisa

Em análise aos indicadores de qualidade das mensurações (Tabela 07), tem-se que todos os construtos alcançaram os patamares exigidos tanto de confiabilidade composta, quanto de variância média extraída (AVE). Conforme Ringle, Silva e Bido (2014), a confiabilidade composta deve ser igual ou superior à 0,7 para garantir o alcance da consistência interna dos construtos. Já a variância média extraída deve alcançar índice igual ou superior à 0,5 , para confirmar a validade convergente dos itens em relação ao seu respectivo construto.

Seguindo as orientações dos autores, para avaliar a validade discriminante adotou-se o critério de Fornell-Larcker (Tabela 08), que prevê que o quadrado das AVE's de cada construto deve ser maior do que seu coeficiente de correlação com os demais construtos que compõem o modelo (Ringle, Silva \& Bido, 2014).

Tabela 07

Validade Discriminante - Critério Fornell-Larcker

\begin{tabular}{l|c|c|c|c|c|c|c|c}
\hline & 1 & 2 & 3 & 4 & 5 & 6 & 7 & 8 \\
\hline 1- Beneficiamento Ativo Ilegal & 0,795 & & & & & & & \\
\hline 2- Beneficiamento Ativo Legal & 0,394 & 0,783 & & & & & & \\
\hline 3- Beneficiamento Passivo & 0,431 & 0,496 & 0,722 & & & & & \\
\hline 4- Beneficiamento Sem Danos & 0,029 & 0,157 & 0,229 & 0,841 & & & & \\
\hline 5- Downloading e Compra de Falsificados & 0,328 & 0,397 & 0,520 & 0,129 & 0,775 & & & \\
\hline 6- Racionalidade Deontológica & $-0,139$ & $-0,171$ & $-0,183$ & $-0,041$ & $-0,188$ & 0,761 & & \\
\hline 7- Propensão ao Consumo de Falsificados & 0,199 & 0,363 & 0,319 & 0,098 & 0,580 & $-0,147$ & 0,752 & \\
\hline 8- Racionalidade Teleológica & 0,149 & 0,170 & 0,114 & $-0,040$ & 0,102 & 0,007 & 0,153 & 0,712 \\
\hline
\end{tabular}

Fonte: Dados da pesquisa

Em análise à Tabela 08, verifica-se que a análise discriminante foi alcançada em conformidade com o critério Fornell-Larcker, o que garante que os construtos que compõem o modelo efetivamente se diferenciam entre si. Sendo assim, uma vez validadas as mensurações realizadas, foi possível seguir adiante com as análises dos dados.

\section{Dimensões éticas da escala de Vitell e Muncy (2005)}

Considerando as proposições de Vitell e Muncy (2005) e os estudos empíricos que utilizaram sua escala de ética do consumidor, tem-se que a análise das pontuações médias obtidas em cada dimensão podem ser usadas como elementos de validação externa, tanto 
para tal teoria, quanto para o estudo em questão. Sendo assim, coerente com os resultados de estudos anteriores (Vitell \& Muncy, 1992; Casali et al., 2010), os dados levantados apontam para o mesmo ordenamento entre as quatro dimensões de comportamentos eticamente questionáveis que compõem a escala de ética do consumidor (Gráfico 02).

Considerando a dimensão Downloading e Compra de Falsificados (DCF), a média de 3,52 pontos revela que os entrevistados tendem a considerar tais comportamentos como sendo "mais errados" ou "menos corretos" do que aqueles que não geram danos diretos a nenhum público (BSD), assim como "menos errados" ou "mais corretos" dos que os comportamentos de beneficiamento passivo (BP). Tais resultados se mostram coerentes com os achados de Vitell e Muncy (2005) e de Casali et al., (2010).

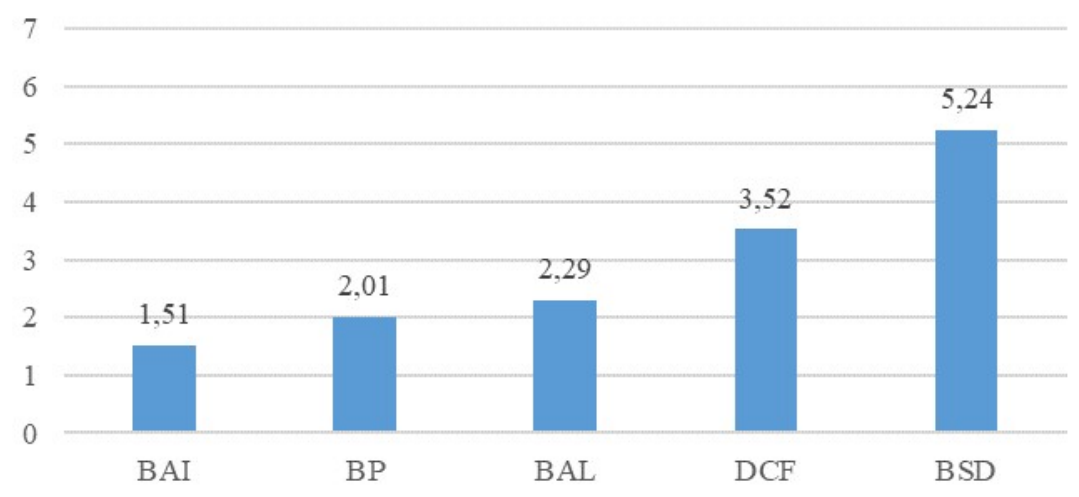

Fonte: Dados da Pesquisa

Gráfico 02: Média das dimensões da escala de ética do consumidor

Aprofundando mais na análise de tais dados, entende-se que o consumo de falsificados é considerado como "menos errado" que os comportamentos de beneficiamento passivo (BP), por ser uma prática socialmente disseminada, não percebida como ilegal. Além disto, por ser considerado "menos correto" do que os comportamentos que não geram danos (BSD), pode-se inferir que, em geral, os consumidores percebem alguma consequência negativa oriunda da compra de falsificados.

\section{Avaliação dos modelos estruturais}

Conforme mencionado anteriormente, para verificação dos demais objetivos adotou-se a técnica de Modelagem de Equações Estruturais. Sendo assim, a Tabela 09 apresenta os coeficientes de caminho (CC), o nível de significância encontrado (Sig), a capacidade explicativa das variáveis independentes em relação à variável dependente $\left(R^{2}\right)$ e os indicadores de qualidade dos modelos (GoF e SRMR). No que se refere aos coeficientes de caminho, Ringle, Silva e Bido (2014) afirmam que não há valores referenciais, sendo sua análise feita à luz da teoria e do objeto de estudo.

Por se tratar de um estudo das ciências sociais, adotou-se o nível de confiança de 95\%, sendo aceitas, com ressalvas, relações que se mostraram significativas ao nível de confiança de até $90 \%$. A análise do $\mathrm{R}^{2}$ considerou que efeitos entre 0,02 e 0,15 são considerados pequenos, entre 0,15 e 0,35 são considerados médios e acima deste valor, são tomados como grandes. Para avaliação do GoF, considerando as divergências ainda existentes na literatura, optou-se por tomá-lo como um indicador de qualidade positivo para efeito de comparação entre os modelos A e B. Sendo assim, quanto maior o GoF, melhor é o ajuste do modelo. Por fim, seguindo as orientações de Hair et al. (2009) optou-se por utilizar mais de um indicador de qualidade dos modelos, sendo desejável ao menos um indicador negativo. Assim, optou-se pela análise do SRMR, que segundo os autores deve ser inferior a 0,08. 
Tabela 09

Avaliação dos modelos estruturais

\begin{tabular}{|c|c|c|c|c|c|c|c|}
\hline Modelo & Relação & $\mathrm{CC}$ & Sig & Hipótese & Status & $\mathbf{R}^{2}$ & $\begin{array}{l}\text { GOF / } \\
\text { SRMR }\end{array}$ \\
\hline \multirow{11}{*}{ A } & Idade $->$ PCF & $-0,017$ & 0,757 & $\mathrm{H} 1$ & Rejeitada & \multirow{11}{*}{$43,9 \%$} & \multirow{11}{*}{$50,9 \%$} \\
\hline & Sexo -> PCF & 0,222 & $0,000^{*}$ & $\mathrm{H} 2$ & Suportada & & \\
\hline & Escolaridade $->$ PCF & 0,027 & 0,665 & H3 & Rejeitada & & \\
\hline & Renda -> PCF & $-0,161$ & $0,007^{*}$ & $\mathrm{H} 4$ & Suportada & & \\
\hline & Deontológico -> PCF & 0,001 & 0,992 & $\mathrm{H} 5$ & Rejeitada & & \\
\hline & Teleológico -> PCF & 0,093 & 0,109 & H6 & Rejeitada & & \\
\hline & BAI -> PCF & $-0,006$ & 0,925 & H8 & Rejeitada & & \\
\hline & BP -> PCF & $-0,025$ & 0,745 & H9 & Rejeitada & & \\
\hline & BAL -> PCF & 0,129 & $0,054^{* *}$ & $\mathrm{H} 10$ & Suportada & & \\
\hline & BSD -> PCF & 0,022 & 0,710 & H11 & Rejeitada & & \\
\hline & DCF $->$ PCF & 0,519 & $0,000^{*}$ & H12 & Suportada & & \\
\hline \multirow{7}{*}{ B } & Idade $->$ PCF & $-0,028$ & 0,612 & $\mathrm{H} 1$ & Rejeitada & \multirow{7}{*}{$42,1 \%$} & \multirow{7}{*}{$\begin{array}{l}50,9 \% \\
48,0 \%\end{array}$} \\
\hline & Sexo -> PCF & 0,233 & $0,000^{*}$ & $\mathrm{H} 2$ & Suportada & & \\
\hline & Escolaridade -> PCF & 0,043 & 0,473 & H3 & Rejeitada & & \\
\hline & Renda -> PCF & $-0,162$ & $0,001^{*}$ & $\mathrm{H} 4$ & Suportada & & \\
\hline & Deontológico -> PCF & 0,006 & 0,917 & $\mathrm{H} 5$ & Rejeitada & & \\
\hline & Teleológico -> PCF & 0,105 & $0,073^{* *}$ & H6 & Suportada & & \\
\hline & Sensibilidade Ética -> PCF & 0,556 & $0,000^{*}$ & $\mathrm{H7}$ & Suportada & & \\
\hline
\end{tabular}

Fonte: Dados da pesquisa

Ao analisar os modelos estruturais (Tabela 09) verifica-se que as relações entre a idade e o nível de escolaridade e a propensão ao consumo de falsificados que haviam sido confirmadas por meio dos testes de comparação de média, não podem ser consideradas significativas por meio da técnica de Modelagem de Equações Estruturais pela abordagem dos Mínimos Quadrados Parciais. Contudo, as relações entre a propensão ao consumo de falsificados e o sexo e a faixa de renda familiar do consumidor se mostram significativas em ambos os modelos.

Sobre as dimensões éticas de consumo (Modelo A), observa-se que somente as dimensões Beneficiamento Ativo Legal (BAL) e Downloading e Compra de Falsificados (DCF) influenciam de modo significativo a propensão ao consumo de falsificados, confirmando as hipóteses H10 e H12. Já no que se refere à sensibilidade ética (Modelo B), os resultados apontam para a existência de uma relação significativa entre tal construto e a propensão ao consumo de falsificados, o que confirma a hipótese H7.

Ainda em análise à Tabela 07 , verifica-se que as racionalidades deontológicas e teleológicas não apresentaram relações significativas com a propensão ao consumo de falsificados em nenhum dos modelos testados, a um nível de confiança de 95\%. Considerando o nível de confiança de $90 \%$, somente a racionalidade teleológica apresentou relação significativa com a propensão ao consumo de falsificados, considerando apenas o Modelo B. Sendo assim, considera-se que há evidencias suficientes para a rejeição das hipóteses H5 e H6.

Visando a proposição de um modelo teórico que explique a propensão ao consumo de falsificados a partir da perspectiva da ética e das características demográficas dos consumidores (Objetivo 4), procedeu-se a análise do $R^{2}$, do GoF's e do SRMR de ambos os modelos. Os resultados apontam para a adoção do modelo A, uma vez que sua capacidade explicativa e seu GoF se mostram superiores aos do modelo concorrente. Além disto, enquanto o SRMR de 0,084 pode ser tolerado (Hair et al., 2009), um índice de 0,090 aponta para a baixa qualidade do modelo B e, portanto, para o seu descarte.

\section{CONSIDERAÇÕES FINAIS}

Visando explicar os elementos que influenciam o consumo de produtos falsificados, o presente estudo confirmou que tanto as características demográficas quanto a ética do consumidor influenciam a propensão ao consumo de falsificados.

A partir dos resultados é possível fazer as seguintes afirmativas em relação à propensão ao consumo de falsificados: a) 0 sexo do consumidor influencia a propensão ao consumo de falsificados. Mulheres apresentam maior propensão a tal comportamento do que os homens; b) Quanto maior a renda familiar do 
consumidor, menor tende a ser a propensão ao consumo de produtos falsificados; c) Quanto mais o consumidor acredita que os comportamentos classificados como "beneficiamento ativo legal" (BAL) não são errados, maior é a propensão ao consumo de falsificados; d) Quanto mais o consumidor acredita que os comportamentos classificados como "download e compra de falsificados" não são errados, maior tende a ser a propensão ao consumo de falsificados; e) Quanto menor a sensibilidade ética do consumidor, maior é a propensão ao consumo de falsificados.

No que se refere às características demográficas, identificou-se que há diferenças significativas na média de propensão ao consumo de falsificados, quando se compara os consumidores estratificados por sexo, idade, renda familiar e escolaridade. Contudo, dada a ausência de relações significativas da idade e da escolaridade em relação à propensão ao consumo de falsificados, tem-se que a confirmação da influência de tais variáveis em relação aos elementos relativos ao consumo de produtos falsificados ainda carece de maior escrutínio e validação externa.

Considerando a ausência de evidências que confirmam a influência das racionalidades éticas do consumidor em relação à propensão ao consumo de falsificados, sugere-se que novos empreendimentos científicos se debrucem na análise de como tais racionalidades afetam outros tipos de comportamentos de consumo que possam envolver algum tipo de componente ético.

Tomando-se por base as proposições de Hunt e Vitell (2006), considera-se relevante o desenvolvimento de procedimentos que permitam avaliar a influência conjunta de ambas as racionalidades éticas e não de cada uma isoladamente, como se fez no presente estudo. Além disto, em coerência com tais autores, faz-se necessário avaliar a sensibilidade dos consumidores em relação aos falsificados, antes de verificar se tais variáveis influenciam ou não a propensão ao seu consumo. Considerando que os elementos éticos somente teriam influência em situações em que os consumidores percebem algum dilema ético (Hunt \& Vitell, 2006), a exclusão dos consumidores com baixa sensibilidade ética para o consumo de falsificados poderia revelar a influência das racionalidades éticas em relação à propensão ao consumo de tais produtos?

Como limitações do estudo, considera-se o uso de uma amostragem não probabilística e sua aplicação a um universo restrito à Região Metropolitana de Belo Horizonte. Além disto, verifica-se que para uma explicação mais abrangente sobre o fenômeno de consumo de falsificados, faz-se necessária integração do framework teórico amparado na ética com os demais elementos já discutidos pela literatura sobre o tema (Eisend \& Schuchert-Güler, 2006; Lee \& Yoo, 2009; Staake, Thiesse \& Fleisch, 2009).

Em suma, no que se refere ao avanço do conhecimento sobre o tema, tem-se que o estudo em tela contribuiu para o entendimento de elementos relacionados ao consumo de falsificados, especificamente no que tange à ética dos consumidores e às características demográficas. Sobre as contribuições gerenciais, além de identificar os consumidores com maior ou menor propensão ao consumo de falsificados, o presente estudo revelou que apesar da uma baixa propensão ao consumo de falsificados (geral), as percepções éticas dos consumidores em relação a tal comportamento estão balizadas entre a falta de percepção da ilegalidade envolvida neste ato e a consciência de suas consequências negativas. Sendo assim, para combate a tal comportamento, além da explicitação das consequências maléficas relacionadas ao consumo de falsificados, sugere-se que empresas, entidades públicas e órgãos de defesa do consumidor enfatizem o caráter ilegal não só de sua produção e distribuição, mas principalmente de sua compra e consumo.

\section{REFERÊNCIAS}

Andrade, M. L., Leite, R. S. \& Salvador, S. (2018) Falsificado sim, mas de coração!: Uma investigação interpretativa sobre $\mathrm{o}$ ato de presentear com produtos falsificados. In Pinto, M. R.; Batinga, G. L. (Org.). Cultura e Consumo no Brasil: estado atual e novas perspectivas. Editora PUC-MG

Andrade, M. L., Leite, R. S., Diniz, W. V., Costa, K. C. \& Ibrahim, S. T. C. A. (2017). Processos Axiológicos: Proposta de (Re)Integração das Teorias Sobre Ética e Valores Pessoais. Anais do XX Seminários em Administração (SEMEAD $X X)$, São Paulo-SP. 
Burns, J. O. \& Kiecker, P. (1995). Tax practitioner ethics: An empirical investigation of organizational consequences. The Journal of the American Taxation Association, 17(2), 20.

Burell, G., \& Morgan, G. (1979). Sociological paradigms and organisational analysis. Elements of the sociology of corporate life.

Calvosa, M. V. D. (2012). Uma pesquisa bibliométrica sobre valores pessoais: a análise global de instrumentos de mensuração de valores pessoais. Anais do XXVII Simpósio de Gestão de Inovação Tecnológica, Salvador-BA.

Casali, R. R. B., Paes, T. A. A., Machado, P. A., Medeiros, L. C \& Aragão, A. J. (2010). Pirataria de Software: Uma Análise da Relação entre Comportamento Ético, Atitude e Intenção do Consumidor. Anais do XXIV Encontro da $A N P A D$, Rio de Janeiro- RJ.

Clavo, L. C. (2008). Aristóteles para Executivos: como a filosofia ajuda na gestão empresarial. São Paulo: Globo.

Conselho Nacional de Combate à Pirataria (CNPC). Brasil Original. Brasília (2011). Disponível em: http://portal.mj.gov.br/main.asp?Team =\%7B 1450A8F5-90F9-4EE7-B58C

36EA406FD360\%7D. Acesso em: 06/09/2014 (17:01)

Costa, F. D. (2011). Mensuração e desenvolvimento de escalas: aplicações em administração. Rio de Janeiro: Ciência Moderna.

Costa, R., \& Sant'Anna, S. R. (2008). O "Legal" do Pirata: Um Olhar Sobre as Práticas de Consumo de Produtos Falsificados. Anais do IV Encontro de Marketing da ANPAD, Curitiba, PR, Brasil, p. 1- 16.

Diniz, W. V., Leite, R. S. \& Pinto, M. R. (2017). Mapeamento Meta-Narrativo da Produção Acadêmica Brasileira Sobre Falsificação. Revista Eletrônica de Administração (REAd), Ed. 86, № 2, Mai-Ago 2017, p. 135-166.

Eisend, M., \& Schuchert-Güler, P. (2006). Explaining counterfeit purchases: A review and preview.Academy of Marketing Science Review, 2006, 1.

Fullerton, S., Kerch, K. B. \& Dodge, H. R. (1996). "Consumer ethics: An assessment of individual behaviour in the marketplace", Journal of Business Ethics, 15, 805-814.

Furnham, A., \& Valgeirsson, H. (2007). The effect of life values and materialism on buying counterfeit products. The Journal of SocioEconomics, 36(5), 677-685.

Gupta, P. B., Gould, S. J., \& Pola, B. (2004). "To pirate or not to pirate": A comparative study of the ethical versus other influences on the consumer's software acquisition-mode decision. Journal of Business Ethics, 55(3), 255274.
Hunt, S. D., \& Vitell, S. J. (2006). The general theory of marketing ethics: A revision and three questions. Journal of Macromarketing, 26(2), 143-153.

Lau, K. W. (2007). Interaction effects in software piracy. Business Ethics: A European Review, 16(1), 34-47.

Lee, S. H. \& Yoo, B. (2009). A review of determinants of counterfeiting and piracy and the proposition for future research. Korean Journal of Policy Studies, 24(1), 1-38.

Malhotra, N. k. \& Birks, D. F. (2007). Marketing Research: An Apllied Approach. 3aㅡ ed. Harlow, Prentice Hall.

Morin, E. (2007). O Método: Ética. Tradução: Juremir Machado Da Silva. 3. Ed. Porto Alegre: Sulina.

Nill, A., \& Shultz, C. J. (1996). The scourge of global counterfeiting. Business Horizons, 39(6), $37-$ 42.

Nunnaly, J. C. \& Bernstein, I. H. (1994). Psychometric Theory. 3a-Ed. McGraw-Hill.

OECD/EUIPO (2016). Trade in Counterfeit and Pirated Goods: Mapping the Economic Impact, OECD Publishing, Paris.

Passos, E. (2006). Ética nas organizações. Editora: Atlas.

Patrus-Pena, R. (2012). Ética e felicidade. Editora Vozes.

Penz, E., \& Stottinger, B. (2005). Forget the 'real' thing - Take the copy! An explanatory model for the volitional purchase of counterfeit products. Advances in Consumer Research, 32(1), 568-76.

Podsakoff, P. M., MacKenzie, S. B., Lee, J-Y, \& Podsakoff, N. P. (2003). Common method biases in behavioral research: a critical review of the literature and recommended remedies. Journal of Applied Psychology, 88(5), 879-903.

Prendergast, G., Hing-Chuen, L., \& Phau, I. (2002). Understanding consumer demand for nondeceptive pirated brands. Marketing Intelligence \& Planning, 20(7), 405-416.

Rabello, R. F., Leite, R. S., \& Gonçalves Filho, C. (2016) Consumo de Falsificados: Analisando a Influência da Ética e dos Riscos. Anais do XL ENANPAD, Costa do Sauipe - BA.

Ringle, C. M., Da Silva, D., \& Bido, D. D. S. (2014). Modelagem de equações estruturais com utilização do SmartPLS. REMark, 13(2), 54.

Ringle, C. M., Wende, S., \& Becker, J.-M. (2015). "SmartPLS 3." Boenningstedt: SmartPLS GmbH, http://www.smartpls.com.

Santos, M. F., Gonçalves-Filho, C. \& Falce, J. L (2017). Entendendo a Compra de Produtos Piratas: a Influência das Estratégias de Negação e Ética do Consumidor. Revista Brasileira de Pesquisas de Marketing, Opinião Pública e Mídia (PMKT), 10(2), 239-265. 
Schwartz, S. H., Cieciuch, J., Vecchione, M., Davidov, E., Fischer, R., Beierlein, C., ... \& Dirilen-Gumus, 0. (2012). Refining the theory of basic individual values. Journal of Personality and Social Psychology, 103(4), 663.

Solomon, S. L., \& O'Brien, J. A. (1990). The effect of demographic factors on attitudes toward software piracy. Journal of Computer Information Systems, 30(3), 40-46.

Staake, T., Thiesse, F. \& Fleisch, E. (2009). The emergence of counterfeit trade: a literature review. European Journal of Marketing, 43(3/4), 320-349.

Swami, V., Chamorro-Premuzic, T., \& Furnham, A. (2009). Faking it: Personality and individual difference predictors of willingness to buy counterfeit goods. The Journal of SocioEconomics, 38(5), 820-825.

Tan, B. (2002). Understanding consumer ethical decision making with respect to purchase of pirated software.Journal of Consumer Marketing, 19(2), 96-111.

Vazquez, A. S. (2007) Ética. 29a ed. Rio de Janeiro: Civilização Brasileira.

Vitell, S. J. (2003). Consumer ethics research: Review, synthesis and suggestions for the future. Journal of Business Ethics, 43(1-2), 3347.

Vitell, S. J., \& Muncy, J. (1992). Consumer ethics: An empirical investigation of factors influencing ethical judgments of the final consumer. Journal of Business Ethics, 11(8), 585-597.

Vitell, S. J., \& Muncy, J. (2005). The Muncy-Vitell consumer ethics scale: A modification and application. Journal of Business Ethics, 62(3), 267-275.

Zimmerman, A., \& Chaudhry, P. (2009). The Economics of Counterfeit Trade: Governments, Consumers, Pirates and Intellectual Property Rights. Springer-Verlag Berlin Heidelberg.

\title{
Ethics and the Consumption of Counterfeits
}

\begin{abstract}
The consumption of counterfeits is a global phenomenon of interest to several researchers in the field of marketing. The present study aimed to explain the elements that influence the propensity to consumption of counterfeits, based on the demographic and ethical characteristics of the consumer. A survey was carried out with a sample of two hundred and three consumers from Regiao Metropolitana de Belo Horizonte. The results indicate that among the demographic characteristics evaluated, gender and family income influence the propensity to consume counterfeit. Women and consumers with lower family income are more prone to such behavior. Regarding the influence of ethics, the results indicate that consumers who are more tolerant of legal consumer practices, even if ethically questionable, are more likely to use counterfeit. Thus, it is suggested that campaigns that demonstrate that the purchase of counterfeits is illegal may contribute to the reduction of this behavior.
\end{abstract}

Keywords: Consumption of Counterfeit; Ethics in Marketing; Consumer Ethics

\section{Apêndice}

Apêndice 1: Escalas que compõem o instrumento de coleta de dados

\begin{tabular}{c|l|l|c|c}
\hline Construto & Dimensão & \multicolumn{1}{|c|}{ Item } & E-M \\
\hline & & $\begin{array}{l}\text { É importante fazer a coisa certa, mesmo que eu ou alguém próximo a } \\
\text { mim sofra muito com as consequências }\end{array}$ & $\mathrm{M}$ \\
\cline { 3 - 4 } $\begin{array}{c}\text { Racionalidade } \\
\text { ética }\end{array}$ & $\begin{array}{l}\text { Eu não preciso conhecer as consequências de uma conduta para dizer se } \\
\text { ela é certa ou errada }\end{array}$ & $\mathrm{E}$ \\
\cline { 3 - 5 } & $\begin{array}{l}\text { É errado decidir o que fazer pensando apenas no equilíbrio entre suas } \\
\text { consequências positivas e negativas }\end{array}$ & $\mathrm{E}$ \\
\cline { 3 - 5 } & $\begin{array}{l}\text { Alguns atos são simplesmente errados, mesmo que possam resultar em } \\
\text { consequências positivas para muitas pessoas }\end{array}$ & $\mathrm{M}$ \\
\cline { 3 - 5 } & $\begin{array}{l}\text { Há importantes regras que determinam se um ato é certo ou errado, } \\
\text { independente das suas consequências }\end{array}$ & $\mathrm{E}$ \\
\cline { 3 - 5 } & Independente das consequências, são os valores morais que determinam & $\mathrm{E}$ \\
\hline
\end{tabular}




\begin{tabular}{|c|c|c|c|}
\hline & & é ético ou antiético & \\
\hline & \multirow{5}{*}{ Teleológica } & $\begin{array}{l}\text { Não é possível determinar o quanto correta ou errada é uma ação sem } \\
\text { considerar suas consequências }\end{array}$ & M \\
\hline & & $\begin{array}{l}\text { Uma ação é correta quando gera muitos benefícios para um grande } \\
\text { número de pessoas }\end{array}$ & M \\
\hline & & $\begin{array}{l}\text { Uma ação é correta quando gera muitos benefícios para mim, minha } \\
\text { família e aqueles com os quais eu me preocupo }\end{array}$ & $\mathrm{E}$ \\
\hline & & $\begin{array}{l}\text { Quanto maior é a probabilidade de consequências positivas resultarem } \\
\text { de uma ação, maior é a certeza de que esta é a coisa certa a ser feita }\end{array}$ & M \\
\hline & & $\begin{array}{l}\text { Independente dos valores morais, o que determina se uma ação é correta } \\
\text { ou errada são suas consequências }\end{array}$ & $\mathrm{E}$ \\
\hline \multirow{7}{*}{$\begin{array}{l}\text { Propensão ao } \\
\text { consumo de } \\
\text { falsificados }\end{array}$} & \multirow{3}{*}{ Pirataria } & DVD Pirata & $\mathrm{M}$ \\
\hline & & Software Pirata & $\mathrm{E}$ \\
\hline & & CD de Música Pirata & M \\
\hline & \multirow{4}{*}{ Falsificação } & Roupa Falsificada & $\mathrm{M}$ \\
\hline & & Relógio Falsificado & M \\
\hline & & Perfume Falsificado & $\mathrm{E}$ \\
\hline & & Óculos Escuros Falsificados & $\mathrm{E}$ \\
\hline \multirow{23}{*}{$\begin{array}{c}\text { Ética do } \\
\text { consumidor }\end{array}$} & \multirow{5}{*}{ BAI } & Devolver bens danificados quando quem danificou foi você. & M \\
\hline & & $\begin{array}{l}\text { Fornecer informações enganosas para um funcionário sobre um item } \\
\text { sem etiqueta de preço. }\end{array}$ & $\mathrm{E}$ \\
\hline & & $\begin{array}{l}\text { Beber uma latinha de refrigerante em um estabelecimento comercial sem } \\
\text { pagar por ele. }\end{array}$ & $\mathrm{E}$ \\
\hline & & $\begin{array}{l}\text { Relatar um item perdido como "roubado" para uma companhia de } \\
\text { seguros com o objetivo de receber o seguro. }\end{array}$ & M \\
\hline & & Usar um código de acesso de longa distância que não lhe pertence. & $\mathrm{E}$ \\
\hline & \multirow{6}{*}{ BP } & $\begin{array}{l}\text { Mudar-se para uma residência, descobrir que a TV a cabo ainda está } \\
\text { conectada e usá-la sem pagar pelo serviço. }\end{array}$ & M \\
\hline & & Mentir sobre a idade de uma criança para obter um preço menor. & M \\
\hline & & Não dizer nada quando um garçom calcular a conta errado a seu favor. & E \\
\hline & & Receber troco a mais e não dizer nada. & E \\
\hline & & Ver alguém furtando artigos de uma loja e não dizer nada. & E \\
\hline & & $\begin{array}{l}\text { Juntar-se a um clube de CDs para receber CDs grátis sem a intenção de } \\
\text { comprar qualquer um. }\end{array}$ & M \\
\hline & \multirow{5}{*}{ BAL } & $\begin{array}{l}\text { Devolver mercadoria para uma loja afirmando que era um presente } \\
\text { quando não era. }\end{array}$ & M \\
\hline & & Não falar a verdade quando negociando o preço de um novo automóvel. & $\mathrm{E}$ \\
\hline & & Usar um cupom com a validade vencida para mercadorias. & E \\
\hline & & Usar um cupom para uma mercadoria que você não comprou. & E \\
\hline & & Mascarar a verdade em uma devolução do imposto de renda. & $\mathrm{M}$ \\
\hline & \multirow{3}{*}{ BSD } & Devolver uma mercadoria depois de comprá-la porque não gostou dela. & $\mathrm{M}$ \\
\hline & & Gravar um filme direto da televisão. & E \\
\hline & & Gastar mais de uma hora provando roupas e não comprar nada. & $\mathrm{M}$ \\
\hline & \multirow{4}{*}{ DCF } & Instalar um software pago em seu computador sem pagar por ele.* & $\mathrm{M}$ \\
\hline & & Copiar um CD ao invés de comprá-lo.* & $\mathrm{M}$ \\
\hline & & Baixar músicas da internet ao invés de comprá-las. & $\mathrm{M}$ \\
\hline & & $\begin{array}{l}\text { Comprar produtos falsificados ao invés de comprar os produtos } \\
\text { originais. }\end{array}$ & M \\
\hline
\end{tabular}

Fonte: Elaborado pelos autores

E-M: Coluna identifica os itens que foram excluídos (E) do modelo final e os que foram mantidos (M)

*Itens originais que compõem dimensão BSD, mas que neste estudo foram atribuídos à dimensão DCF 